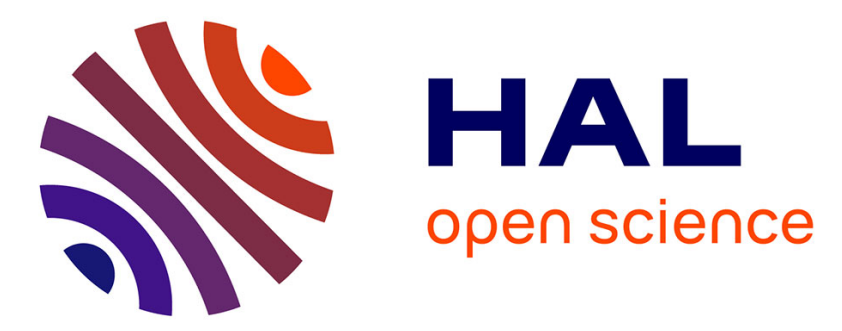

\title{
Imbibition and structure of silica nanoporous media characterized by neutron imaging
}

Marguerite Léang, Frédéric Ott, Frédérique Giorgiutti-Dauphiné, Ludovic Pauchard, Lay-Theng Lee

\section{- To cite this version:}

Marguerite Léang, Frédéric Ott, Frédérique Giorgiutti-Dauphiné, Ludovic Pauchard, Lay-Theng Lee. Imbibition and structure of silica nanoporous media characterized by neutron imaging. Journal of Colloid and Interface Science, 2020, 565, pp.474-482. 10.1016/j.jcis.2020.01.052 . hal-03028852

\section{HAL Id: hal-03028852 \\ https://hal.science/hal-03028852}

Submitted on 7 Oct 2021

HAL is a multi-disciplinary open access archive for the deposit and dissemination of scientific research documents, whether they are published or not. The documents may come from teaching and research institutions in France or abroad, or from public or private research centers.
L'archive ouverte pluridisciplinaire HAL, est destinée au dépôt et à la diffusion de documents scientifiques de niveau recherche, publiés ou non, émanant des établissements d'enseignement et de recherche français ou étrangers, des laboratoires publics ou privés. 


\title{
Imbibition and structure of silica nanoporous media characterized by neutron imaging
}

\author{
Marguerite Léang ${ }^{\mathrm{a}, \mathrm{b}}$, Frédéric Ott ${ }^{\mathrm{a}}$, Frédérique Giorgiutti-Dauphiné ${ }^{\mathrm{b}}$, Ludovic Pauchard ${ }^{\mathrm{b}}$, Lay-Theng Lee ${ }^{\mathrm{a}, *}$ \\ 'Laboratoire Léon Brillouin, UMR12 CEA-CNRS, Université Paris-Saclay, CEA-Saclay, 91191 Gif-sur-Yvette Cedex, France \\ ${ }^{b}$ Laboratoire F.A.S.T., Université Paris-Sud, CNRS, Université Paris-Saclay, F-91405 Orsay, France
}

\section{G R A P H I C A L A B S T R A C T}

Real-time neutron imaging of water imbibition in silica nanoporous media.

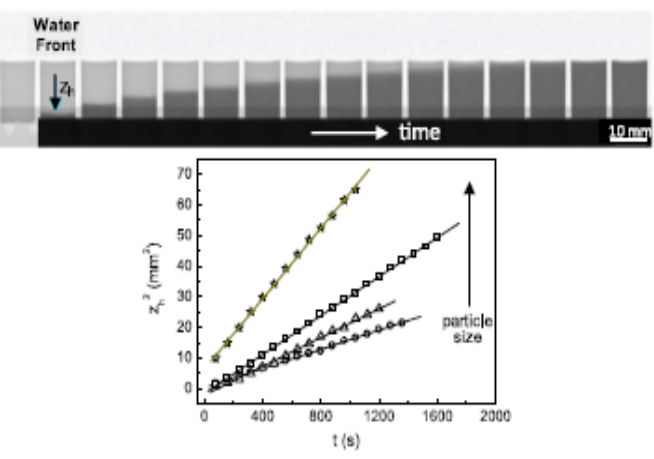

\section{A R T I C L E I N F O}

\section{Article history:}

Received 8 November 2019

Revised 14 January 2020

Accepted 16 January 2020

Available online 17 January 2020

\section{Keywords:}

Neutron imaging

Nanoporous media

Imbibition

Porosity

Permeability

Liquid front broadening

\begin{abstract}
A B S T R A C T
Hypothesis: Colloidal silica dispersions dried under controlled conditions form solid gels that display mechanical properties similar to those observed in several practical processes. An understanding of their structural characteristics and liquid flow properties can therefore help establish these gels as an alternative family of model materials to study practical porous systems.

Experiments: Neutron radiography is a non-destructive technique well-adapted to study hydrogen-rich domains in porous materials due to the high attenuation power of hydrogen. We apply this technique to study gels prepared from silica nanoparticles of radii $5-40 \mathrm{~nm}$.

Findings: The water content in the gels have been quantified and different types of porosities have been determined: total porosity, effective porosity that contributes to liquid flow, and residual porosity that contains bound residual water. This residual water increases with decrease in particle size and constitutes an important fraction of the gel. The dynamics of water imbibition follows a $\sqrt{ } \mathrm{t}$ law, from which the effective pore size and permeability are evaluated. We highlight the role of particle size on water retention, on particle organization and its impact on mechanical resistance. Quantitative analysis of the propagating liquid front shows front broadening that suggests elongated pores with reduced correlated liquid menisci.
\end{abstract}

\footnotetext{
* Corresponding author at: Laboratoire Léon Brillouin, UMR12 CEA-CNRS, Université Paris-Saclay, CEA-Saclay, 91191 Gif-sur-Yvette Cedex, France.

E-mail addresses: leangmarguerite@yahoo.fr (M. Léang), Frederic.Ott@cea.fr (F. Ott), frederique.giorgiutti@u-psud.fr (F. Giorgiutti-Dauphiné), ludovic.pauchard@u-psud.fr (L. Pauchard), Lay-Theng.Lee@cea.fr (L.-T. Lee).
} 


\section{Introduction}

Fluid flow in porous media occurs in numerous processes: in daily activities when food and paper come into contact with liquids; in natural settings as in plants and soils; in industrial and engineered materials for building blocks and foundations as well as for storage; in cultural heritage structures and art paintings. Considerable interests have therefore been vested in understanding this widespread occurrence, namely in the uptake of water in cement and sandstone-based porous media in view of its deleterious effects on structural and mechanical properties of building materials, in the context of geology, and in heritage preservation and restoration. Classic methods such as gravimetry and electrical techniques based on conductivity give information on the water content in the sample. These techniques, however, do not access buried spatial information and the kinetics of the liquid flow front, both of which relate the structural aspects of the porous media. To study internal structures, X-ray tomography has been used to characterize siliceous granular materials but to visualize the liquid phase, it is often necessary to add contrast agents such as metal salts to distinguish between the water and air phase [1-3]. These contrast agents however, can lead to changes in interfacial and wetting properties of the substrate $[4,5]$.

In the past decades, neutron radiography has emerged as a powerful technique to investigate the distribution and flow of fluid in porous structures. The high penetration power of neutron up to several centimeters, its sensitivity to light elements and the large neutron cross-section for hydrogen compared to other heavier elements, render neutron radiography a well-adapted technique to probe water and other hydrogen-rich liquids in porous media. For siliceous porous media, the high scattering power of hydrogen compared to the siliceous material provides high attenuation contrast of the transmitted neutron beam, facilitating the distribution and movement of water in the porous media to be visualized and quantified directly from transmission measurements. Descriptions and reviews of the neutron imaging technique and its applications can be found in the literature [6,7]. To name a few examples, this technique has been applied to studies on theoretical modeling of water [8] and of liquid hydrocarbon [9] imbibition in model mesoporous glass, and of water in granular quartz [10]; to applied systems such as water uptake in natural sandstones [11,12], fractures and water imbibition in sedimentary rock [13], cracks and water uptake in concrete [14], water penetration in different types of porous natural rock stones [15], water transport in low-temperature proton exchange membrane fuel cells $[16,17]$ and water uptake in plants [18]. Furthermore, due to the non-invasive nature of neutron techniques, they have become useful tools for archaeology and cultural heritage research where they are used in combination with other techniques to reveal buried chemical components [19], to study adhesive and filler materials and protective coatings in cultural heritage objects [20], water uptake in historic building materials [21] and moisture retention in painting canvas layers [22].

Neutron radiography is thus a powerful and non-destructive technique that is well suited to probe water content and internal morphology of porous media. In this work, we apply neutron radiography to quantify water content and to track real-time spontaneous water imbibition in porous media prepared by evaporative drying of aqueous dispersions of colloidal silica of different particle sizes. The structural aspects and particle organization for this system during evaporative drying, at the surface and in the bulk, have been investigated in our past studies by neutron reflectivity and small angle neutron scattering [23]. During drying, particle concentration increases until a critical concentration beyond which mechanical stress develops in the wet gel that can lead to crack formation in the solid gel. Interestingly, the crack patterns thus formed show great similarity to those observed in some historic paintings. In our past study, these crack patterns were analyzed and related to mechanical properties using a poroelasticity model that predicts well the behavior of both the silica gel and paint materials used in the historic epoch, thus providing a potential method to evaluate and authenticate paint layers of historic art paintings [24]. In a next study, with a parallel interest in art painting restoration by solvents and possible accompanying deleterious effects, the dynamics of penetration of millimeter-size sessile drops of different solvents into the silica porous media were visualized by optical microscopy and discussed in relation to their impact on mechanical properties [25]. In the present study, neutron radiography is employed to give a complete characterization of evaporation-consolidated silica porous media prepared from different particle sizes. From transmission measurements we quantify the water content in the porous media to evaluate their porosity, distinguishing between "effective porosity" that constitutes pore space available for imbibition and "residual porosity" where the pore space is occupied by residual hydration water. Real-time dynamic transmissions are employed to visualize directly spontaneous capillary imbibition in the Lucas-Washburn regime, to extract information on effective pore size and permeability, and to quantify the width of the propagating liquid front that relates pore morphology.

\section{Experimental section}

\subsection{Sample preparation}

The samples studied were porous media prepared by controlled drying of silica colloidal dispersions. Four different samples were used: Ludox SM30, HS40, TM50 from Sigma-Aldrich, and Klebosol50 from Merck, with initial mass fractions $0.3,0.4,0.5$ and 0.5 respectively. The dispersions, stabilized at initial $\mathrm{pH} 9-10$, were used as received. The particle radius $a$ and log-normal polydispersity $p / a$ characterized by small-angle neutron scattering (SANS) are SM30: $a=4.7 \mathrm{~nm}, p / a=0.20$; HS40: $a=7.9 \mathrm{~nm}, p / a=0.16$; TM50: $a=12.8 \mathrm{~nm}, p / a=0.14$; Klebosol: $a=37.8 \mathrm{~nm}, p / a=0.14$. The dispersions were dried in an enclosed box in the presence of $\mathrm{Mg}$
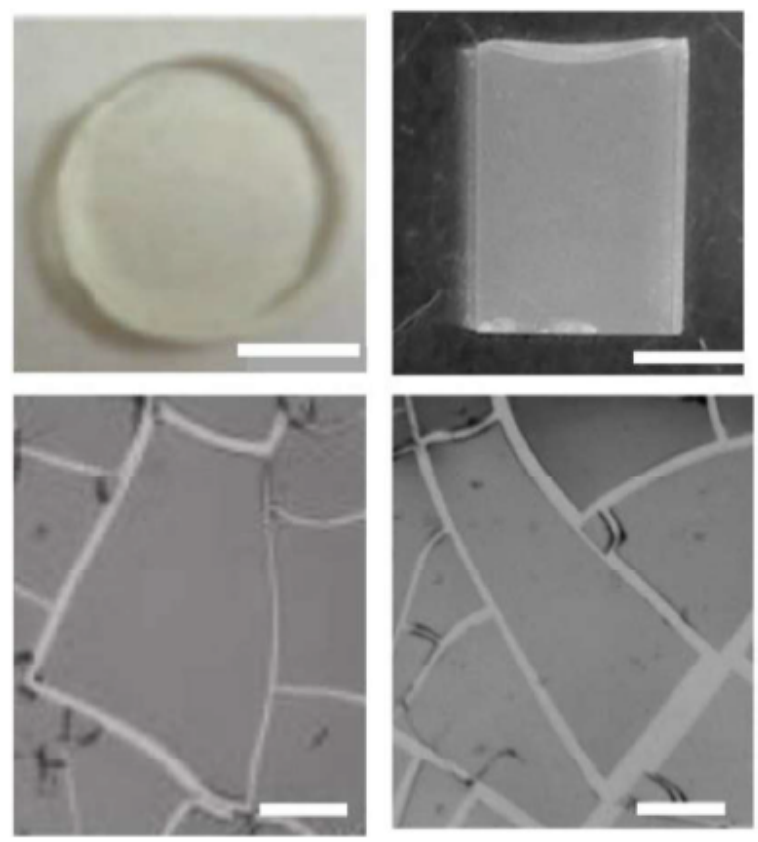

Fig. 1. Porous media samples of different geometries prepared by controlled drying of colloidal silica dispersions: circular and rectangular pastilles (top), fragments dried in a large glass dish (bottom); bar scale $=5 \mathrm{~mm}$. 
$\left(\mathrm{NO}_{3}\right)_{2}$ to control the drying conditions under relative humidity $\mathrm{RH}=52-54 \%$ at $\mathrm{T}=20-23{ }^{\circ} \mathrm{C}$. Under these controlled atmospheric conditions, it was possible to produce sufficiently large samples for the imaging studies. Furthermore, different sample geometries were prepared (Fig. 1): (i) rectangular sample $(2 \mathrm{~cm} \times 1 \mathrm{~cm})$ dried in Teflon containers, (ii) circular pastille $(1 \mathrm{~cm}$ diameter) dried in circular rubber rings on a glass plate, and (iii) "fragments" of dispersions dried in a large glass dish. The sample thicknesses measured with an electronic caliper varied from about 1 to $2 \mathrm{~mm}$.

\subsection{Neutron imaging experimental setup}

Neutron radiography experiments were performed on the neutron imaging spectrometer IMAGINE at the ORPHEE reactor. The neutron beam is polychromatic with a wavelength range of about 3-25 $\AA$ with a peak flux at $4 \AA$ and an integral neutron flux intensity of $2 \times 10^{7} \mathrm{n} \cdot \mathrm{cm}^{-2} \cdot \mathrm{s}^{-1}$. For image detection, a lithium scintillator of thickness $100 \mu \mathrm{m}$ converts gamma rays to photons that are captured indirectly by an SCMOS ANDOR NEO camera via a reflecting mirror to reduce radiation damage to the camera. The samples were clamped vertically by two parallel aluminum bars and placed in an aluminum container bath and the ensemble was positioned as close as possible to the detector to minimize geometric blurring of the image. This blurring effect is defined as: [26] $b_{g}=l /(L / D)$ where $l$ is the sample-detector distance, and $L$ and $D$ are the aperture-detector distance and aperture size, respectively; the ratio $L / D$ is the instrument collimation that is optimized for resolution and beam intensity. The experimental setup is shown in Fig. 2 and the details of the configuration are given in Table 1 . For the imaging measurements, ten dark field images (beam closed, without sample) and ten flat field or incident beam images (beam open, without sample) of $80 \mathrm{~s}$ each were acquired before each series of experiments. For imbibition measurements, the image of the initial "dry" sample was taken, then water was added to the aluminum container and image acquisition was started immediately with an exposure time of $80 \mathrm{~s}$ per image at $80 \mathrm{~s}$ frequency until saturation of the sample.

\subsection{Image processing}

Neutron imaging is based on the transmission of a neutron beam attenuated by a sample according to an exponential attenuation law [27]:

$$
I=I_{0} e^{-\mu_{m} h_{m}}
$$

The transmission is $T=I / I_{0}$ where $I$ and $I_{0}$ are the intensities of the transmitted and incident beams respectively, $\mu_{m}$ is the coefficient of attenuation (scattering + absorption) and $h_{m}$ the thickness

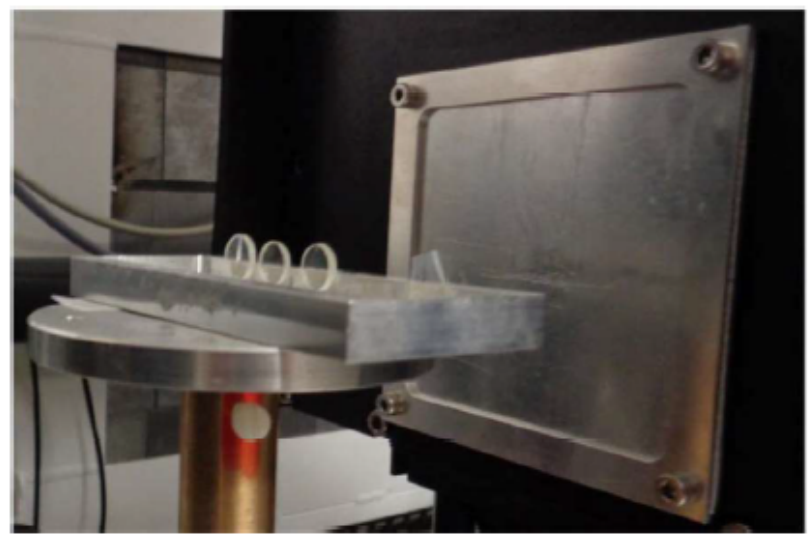

Fig. 2. Setup of the imaging experiments. The samples are clamped vertically between two parallel aluminum bars and place in an aluminum water bath placed close to the detector.
Table 1

Configuration of the neutron imaging setup.

\begin{tabular}{ll}
\hline Aperture diameter, $D$ & $2 \mathrm{~cm}$ \\
Aperture-detector distance, $L$ & $400 \mathrm{~cm}$ \\
Beam divergence, $L / D$ & 200 \\
Field of view & $8 \mathrm{~cm} \times 8 \mathrm{~cm}$ \\
Sample-detector distance, $l$ & $\sim 2 \mathrm{~cm}$ \\
Pixel size & $40 \mu \mathrm{m}$ \\
Spatial resolution/Geometric blurring, $b_{g}=l /(L / D)$ & $\sim 100 \mu \mathrm{m}$ \\
\hline
\end{tabular}

of the material. In order to optimize the image quality and to quantify the transmission, the two-dimensional raw intensity images are corrected using Image J software for background or camera offset by a dark field image, $I_{D F}$ (acquired with closed beam without sample); spatial inhomogeneities of the beam and on the detector are in principal accounted for when the sample image is normalized by the incident beam image. A rescale factor $f$ is also applied to adjust for possible time-fluctuation of the incident beam intensity: $f=I_{\text {ref }} / I_{\text {ref }, 0}$ where $I_{\text {ref }}$ is the average intensity of a reference area on the sample image (outside the sample) and $I_{\text {ref }, 0}$ the average intensity of the same area on the incident beam image. The corrected images are then filtered to remove bright gamma spots (3-5 pixels). This correction permits the homogenization of the gray scale from 0 (black) to 1 (white); black corresponds to attenuated transmission by the water. The final transmission is:

$T=f \frac{i-I_{D F}}{I_{0}-l_{D F}}=e^{-\mu_{m} h_{m}}$

\section{Results and discussions}

\subsection{Static transmission measurements: water content and porosity}

The transmission of a sample depends on its chemical content. For the porous media containing silica and water, the total transmission is given by:

$$
T=e^{-\mu_{m} h_{m}}=e^{-\mu_{\mathrm{SiO}_{2}} h_{\mathrm{SiO}_{2}}}, e^{-\mu_{\mathrm{H}_{2}} \mathrm{O}_{\mathrm{H}_{2} \mathrm{O}}}
$$

where $\mu_{\mathrm{SiO}_{2}}$ and $\mu_{\mathrm{H}_{2} \mathrm{O}}$ are the coefficients of attenuation of silica and water, and $h_{\mathrm{SiO}_{2}}$ and $h_{\mathrm{H}_{2} \mathrm{O}}$ their corresponding effective thicknesses. The thermal neutron cross sections, $b$, for $\mathrm{Si}$ and $\mathrm{O}$ are much smaller than that for $\mathrm{H}[11,28]$, with corresponding scattering (attenuation) power, $b_{\mathrm{SiO}_{2}}=10.64 \times 10^{-24} \mathrm{~cm}^{2}$ and $b_{\mathrm{H}_{2} \mathrm{O}}=167.6 \times 10^{-24} \mathrm{~cm}^{2}$. The transmission attenuation coefficient of silica is thus negligible compared to that of water, and attenuation in transmission can be considered to arise from the water only:

$$
T=e^{-\mu_{\mathrm{H}_{2}} o^{h_{\mathrm{H}_{2} \mathrm{O}}}}
$$

The coefficient of attenuation of water $\mu_{\mathrm{H}_{2} \mathrm{O}}$ is evaluated from a calibration curve with $\mathrm{H}_{2} \mathrm{O}$ contained in quartz cells of known thicknesses. These results are shown in Fig. 3 where a good fit to the transmission for water thickness up to $2 \mathrm{~mm}$ is obtained with $\mu_{\mathrm{H}_{2} \mathrm{O}}=0.45 \mathrm{~mm}^{-1}$. This value pertains to the average for the polychromatic neutron beam and the experimental configuration used in the current study and will be used to evaluate the effective water thickness in the material. Thus, for this silica water system, the total water content is accessible directly from its total transmission. The transmission measurements of the samples were taken before and after filling the container with water and the samples left to imbibe water until complete saturation. In the transmission images, water-rich regions in the sample appear dark due to attenuation by hydrogen in the water; completely wet and saturated sample shows a homogeneously attenuated transmission. Note that the transmission is integrated over the total sample thickness (1-2 mm). For these static measurements, ten images of 


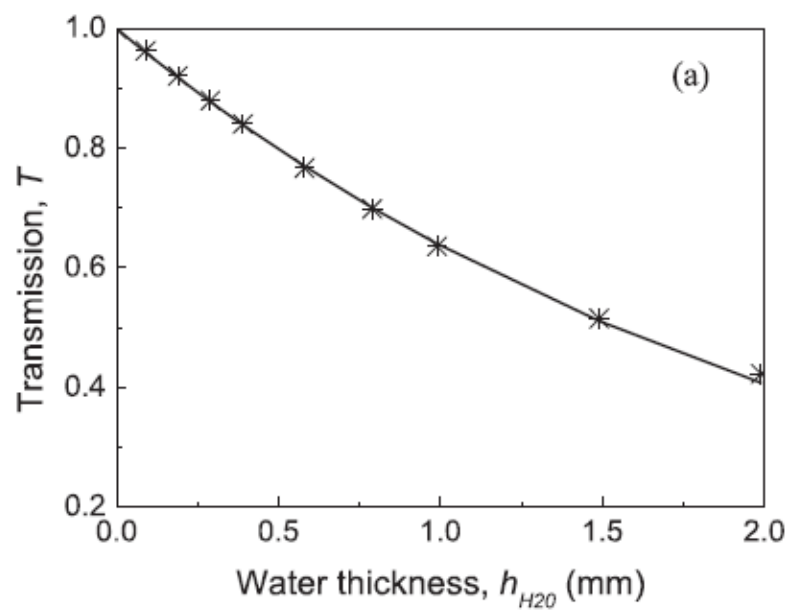

(b)

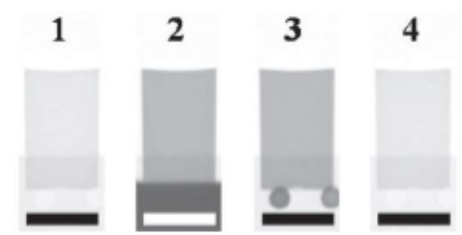

(c)

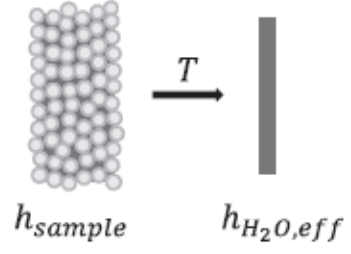

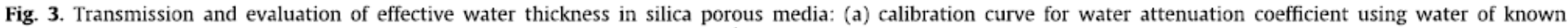

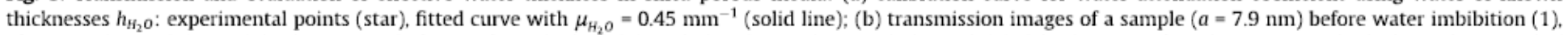

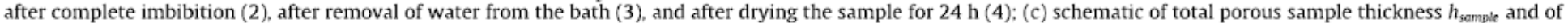
effective water thickness $h_{\mathrm{H}_{2} \mathrm{O} \text {,efj }}$ deduced from the water transmission calibration curve.

$80 \mathrm{~s}$ each are summed for improved statistics to give an accurate measure of the water content. In this case, since the water is distributed throughout the porous sample, the water thickness extracted using the calibration curve corresponds to an effective thickness, $h_{\mathrm{H}_{2} \mathrm{O} \text {,eff }}$ (see schematic in Fig. 3). Dividing $h_{\mathrm{H}_{2} \mathrm{O}, \text { eff }}$ by the total thickness of the porous sample, $h_{\text {sample }}$ measured before imbibition gives directly the sample porosity, $\phi_{\text {pore }}$.

Due to the hydrophilic nature of the material and to the nanosize pores (high capillary pressure), we do not expect wetting problems. Therefore, after complete imbibition, we consider all available pores to be saturated with water. The total water content in the sample constitutes the total volume not occupied by the silica and thus characterizes the total porosity of the sample, $\phi_{\text {pore tot }}$. Importantly, before imbibition, the initial "dry" sample also shows significant attenuation, indicating the presence of non-negligible amount of water. Here, we consider this fraction of water to be stationary and part of the "dry" gel, and the volume occupied by this residual water constitutes a "residual" porosity $\phi_{\text {pore res }}$ that is not available for the flow of imbibed water. Thus two types of pores are evaluated directly from these static transmission measurements, $\phi_{\text {pore,res }}$ and $\phi_{\text {pore,tot }}$ from the initial "dry" sample before imbibition, and the wet sample after complete water imbibition respectively:

Total porosity: $\phi_{\text {pore,tot }}=\frac{h_{\mathrm{H}_{2}, \text { e,ff-wet }}}{h_{\text {sanple }}}$ (from a completely saturated sample after imbibition)

Residual porosity: $\phi_{\text {pore, res }}=\frac{h_{H_{2} 0 \text { eff-res }}}{h_{\text {sample }}}$ (from an initial "dry" sample before imbibition)

Note that $\phi_{\text {pore,res }}$ can also be extracted from a completely saturated sample left to dry for $24 \mathrm{~h}$; these two methods give similar results. From these two types of porosities determined before water contact and after complete water imbibition, we deduce an effective porosity: $\phi_{\text {pore eff }}=\phi_{\text {pore tot }}-\phi_{\text {pore,res }}$ that we consider here to represent the available volume for liquid flow within the porous media, or the fraction of pores that participates in imbibition. Fig. 4 shows the three different types of porosity as a function of particle size, for fragments of samples prepared in a large glass dish. Each point on the graph represents an average from 10 to 15 different fragment samples prepared in different batches at different times, under the same controlled drying conditions. The total porosity $\phi_{\text {pore tot }}$ increases with decrease in particle size, and for all the samples studied here, falls in the range $0.40-0.53$ which is higher than

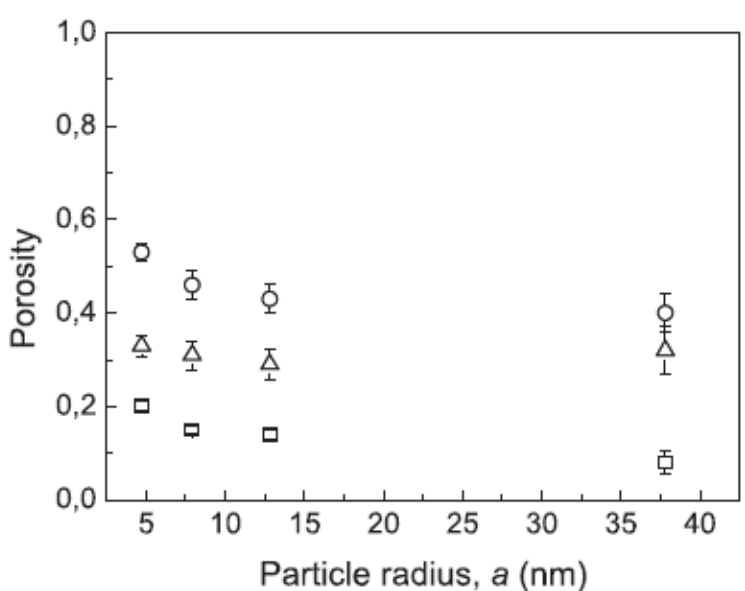

Fig. 4. Different types of porosity versus particle size: residual porosity (square), effective porosity (triangle), total porosity (circle). The samples are fragments from silica dispersions dried in a large glass dish; each point represents an average value from $10-15$ fragments.

the theoretical size-independent value of around 0.36 for dry close-packed spherical particles. (Samples dried at higher humidity, $54 \% \leq \mathrm{RH} \leq 90 \%$, give similar results; on the other hand, those dried at $\mathrm{RH}=13 \%$ are more fragile and prone to fracture into small fragment pieces). The $\phi_{\text {pore,tot }}$ obtained here, defined as the total volume fraction not occupied by silica, are in good agreement with the total silica volume fraction, $\phi_{\mathrm{Si}_{2}, t o t}$ evaluated from our previous SANS measurements [23]. These independent sets of measurements using two separate techniques give: $\phi_{\mathrm{SiO}_{2}, \text { tot }}+\phi_{\text {pore, tot }} \approx 1$. The results are reported in Table 2.

The high $\phi_{\text {pore,tot }}$ obtained for these samples may be attributed to the elevated degree of residual water in the porous media, $\phi_{\mathrm{H}_{2} \mathrm{O} \text {,res }}$. This residual water cannot be removed even after heating at $120^{\circ} \mathrm{C}$ for over a month. It represents hydration water that is chemically bound to the silica surface, in interparticle cohesion bridges or locked in occluded pores. This "trapped" water is an integral component of the solid silica gels consolidated by drying of the colloidal dispersions; it is related to the particle organization and appears to affect particle cohesion and the mechanical properties of the porous media. We note for example that SM30 samples ( $a=4.7 \mathrm{~nm}$ ) contain about $20 \%$ in volume residual water, the particles are less organized as revealed by the broad structure peak in the SANS results (see supplementary information S1), and they 
Table 2

Porosity evaluated from neutron imaging and total particle volume fraction $\phi_{\mathrm{Si}_{2}, \text { tot }}$ measured from SANS [23].

\begin{tabular}{|c|c|c|c|c|}
\hline \multirow[b]{2}{*}{$a(\mathrm{~nm})$} & \multicolumn{3}{|c|}{ Neutron Imaging } & \multirow{2}{*}{$\begin{array}{l}\text { SANS } \\
\phi_{\mathrm{SiO}_{2,} \text { tot }}\end{array}$} \\
\hline & $\phi_{\text {pore, res }}$ & $\phi_{\text {pore }, e f f}$ & $\phi_{\text {pore tot }}$ & \\
\hline 4.7 & $0.20 \pm 0.01$ & $0.33 \pm 0.02$ & $0.53 \pm 0.02$ & $0.36 \pm 0.04$ \\
\hline 7.9 & $0.15 \pm 0.01$ & $0.31 \pm 0.03$ & $0.46 \pm 0.03$ & $0.47 \pm 0.05$ \\
\hline 12.8 & $0.14 \pm 0.01$ & $0.29 \pm 0.03$ & $0.43 \pm 0.03$ & $0.56 \pm 0.05$ \\
\hline 37.8 & $0.08 \pm 0.01$ & $0.32 \pm 0.04$ & $0.40 \pm 0.04$ & - \\
\hline
\end{tabular}

form large and mechanically resistant solid gels. TM50 samples $(a=12.8 \mathrm{~nm})$ contain $10 \%$ in volume residual water with welldefined structure peak and form more brittle samples. Optically, the high water content in SM30 gel renders it transparent while TM50 gel is translucent-white (optical index of silica $=1.44$, water $=1.33$, air $=1.0$ ). Klebosol gels contain only $8 \%$ in volume residual water, the particles are highly organized, and the gels are extremely brittle and fragile and optically white.

\subsection{Dynamic water imbibition measurements: effective pore radius and permeability}

In this section, we address the dynamics of water imbibition in the porous media. The effective porosity $\phi_{\text {pore,eff }}$ evaluated from static measurements is considered here as the fraction of pores that contributes to the capillary water uptake. Dynamic water imbibition measurements were carried out with the samples immersed in a water bath and tracking the real-time propagation of the waterfront. Images of the "dry" samples were first acquired. After the bath was filled with water to the bottom of the samples, time-sequence images were acquired at regular intervals until complete saturation of the sample. The total time of the measurement depends on the height of the sample and varied between approximately 20 to $90 \mathrm{~min}$. Fig. 5 shows the time-sequence transmission images. To illustrate the technique, images for different sample geometries are shown.

Fig. 5a shows images of the total transmission for a rectangular sample. The first image on the left corresponds to the "dry" image before the addition of water to the container and those to its right the time-sequence images after water addition. During imbibition, the strongly attenuated transmission by water-rich domain and the movement of the ascending waterfront are clearly visible. Here, since the initial "dry" sample shows non-negligible attenuation due to residual water, the dark region contains both the residual as well as the imbibed water. Nevertheless, it is possible to visualize attenuation by the imbibed water only, by dividing the image of

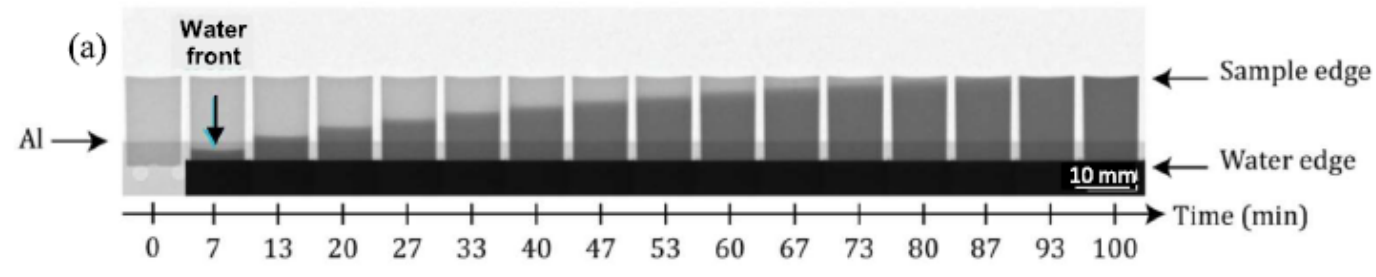

(b)

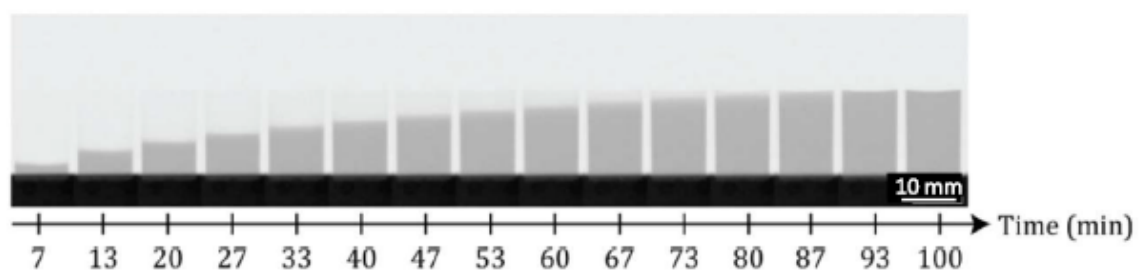

(c)

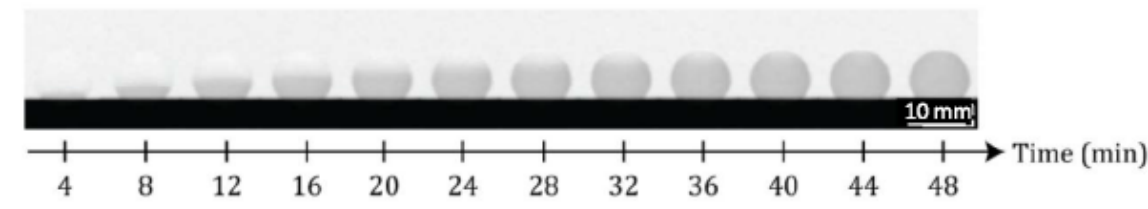

(d)

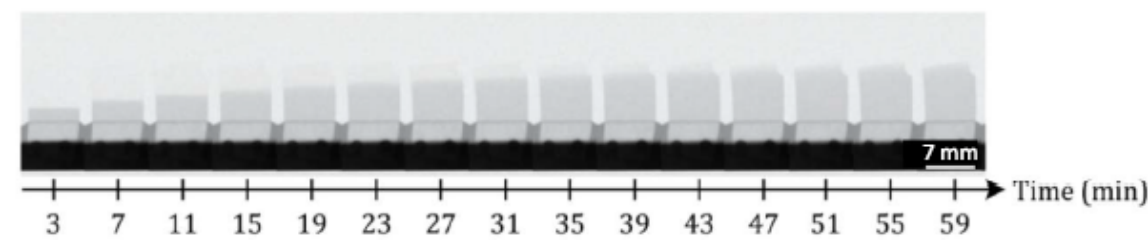

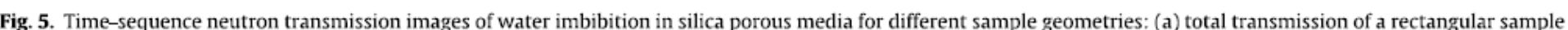

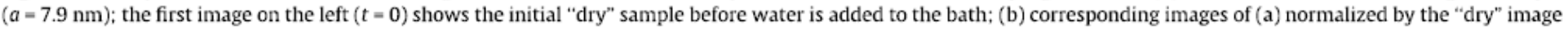

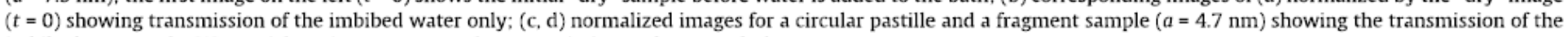
imbibed water only. Water-rich regions attenuate the transmission and appear dark. 
imbibition $\left(T_{t}\right)$ at time $t$ by the initial image before imbibition $\left(T_{0}\right)$. Fig. $5 \mathrm{~b}$ shows the corresponding normalized images of Fig. 5a. In this case, the normalized transmission $\left(T_{\text {norm }}\right)$ is attenuated only by the imbibed water:

$T_{\text {norm }}=\frac{T_{1}}{T_{0}}=\exp \left(-\mu_{\mathrm{H}_{2} \mathrm{O}}\left[h_{\mathrm{H}_{2} \mathrm{O}, \mathrm{t}}-h_{\mathrm{H}_{2} \mathrm{O}, 0}\right]\right)$

where $\left[h_{H_{2} O, t}-h_{H_{2} \mathrm{O}, 0}\right]$ represents the effective thickness of the imbibed water at time $t$. Fig. $5 \mathrm{c}$ shows the normalized images for a circular sample, and Fig. 5d shows normalized images for a fragmented sample. Fragments, as mentioned earlier, are pieces of samples from dispersion dried under controlled conditions in a large glass dish, where the internal drying stress has been released by crack formation. The samples remain intact even after complete water saturation, with no visible swelling (occasional defect and microcrack are immediately detectable as the sample breaks and falls off the sample holder). These experiments performed on different sample geometries demonstrate the direct and non-destructive technique that is well-adapted to study water imbibition in porous media.

In order to quantify the dynamics of imbibition, the gray scale profile along the axis of water propagation is plotted to extract the average water-front position, $z_{h}$. These profiles, along with the corresponding spatiotemporal image, are shown in Fig. 6; the evolution of $z_{h}$ with time can be seen to follow a $\sqrt{t}$ behavior for all the particle sizes.

Liquid flow in porous media traverses different regimes: an inertial regime that is characterized by a linear behavior of the fluid front with time, $z_{h} \sim t$ but this regime occurs in the initial stage of the liquid ascension (first few seconds). This is followed by a viscous regime where for a cylindrical tube, the flow rate per unit area is described by the Hagen-Poiseuille law: $d z / d t=\left(\Delta P R_{\eta}^{2}\right) /(8 \eta z)$ where $\Delta P$ is the pressure drop of the liquid over a distance $z, R_{\eta}$ is the hydraulic radius and $\eta$ the dynamic viscosity. In capillary flow, the viscous drag is balanced by the Laplace capillary pressure: $\Delta P=(2 \gamma \cos \theta) / R_{c}$ where $\gamma$ is interfacial tension between the liquid and air phase, $\theta$ is the contact angle between the liquid and solid phase, and $R_{c}$ the capillary radius. This regime, driven purely by capillary and viscous forces, is the LucasWashburn regime where inertial and gravitational forces are negligible. Gravitational force halts the liquid rise and at equilibrium, the capillary pressure is balanced by the liquid hydrostatic pressure: $\Delta P=(2 \gamma \cos \theta) / R_{c}=\rho g z_{g}$ where $\rho$ is the liquid density, $g$ the gravitational force and $z_{\mathrm{g}}$ the equilibrium liquid height. For water in nanosize pores, $z_{g}$ is several kilometers and is therefore not relevant in the current experiments. Equating the Hagen-Poiseuille and the Laplace pressure, the Lucas-Washburn regime describes the dynamics of capillary liquid rise as follows: $[29,30]$ $z_{h}(t)=\sqrt{\frac{\gamma \cos \theta R_{\eta}^{2}}{2 \eta R_{c}}} \sqrt{t}=\sqrt{\frac{\gamma \cos \theta R_{w}}{2 \eta}} \sqrt{t}$

$z_{h}(t)$ is the average location of the liquid front, and in this study using Millipore water, $\gamma=70 \mathrm{mN} \cdot \mathrm{m}^{-1}, \eta=10^{-3} \mathrm{~Pa} \cdot \mathrm{s}$ at ambient temperature, and assuming total wetting on the hydrophilic silica, $\theta=0^{\circ} . R_{w}=R_{\eta}^{2} / R_{c}$ is the Washburn radius that relates the hydraulic and capillary radii in the viscous and capillary forces respectively. For a system with uniform pore radius, $R_{\eta}=R_{c}$. In practice, however, due to non-uniform pore size and to the different dependencies of the viscous and capillary forces on pore radius, $R_{w}$ tends to indicate a smaller value than the real physical pore size; this disparity has been confirmed by microscopy measurements that show the real pore size to be much larger than $R_{w}$ [31]. To account for pore size dispersion and the complex morphology of the porous media, the Washburn radius has been expressed in terms of a more realistic effective pore radius, $R_{\text {eff }}$ and the tortuosity, $\tau$ of the porous media $[15,32,33]: R_{w}=\frac{R_{\text {eff }}}{\tau^{2}}$. Tortuosity refers to the ratio of the actual path length and the linear length, and direct and independent measurement of its value is not simple. According to theory based on electrokinetic considerations, tortuosity for porous media with spherical particles may be expressed as: [34] $\tau=1 / \sqrt{\phi_{\text {pore,eff }}}$. Using this expression and the $\phi_{\text {pore,eff }}$ evaluated from static transmission measurements, we deduce $\tau=1.7-1.9$, and $R_{\text {eff }}$ that, accordingly, is about three times larger than $R_{\mathrm{w}}$. The dependence of $R_{\mathrm{eff}}$ on particle size $a$ is shown in Fig. 7; for comparison, theoretical curves for the dependence of throat radius on particle size for random and reg-

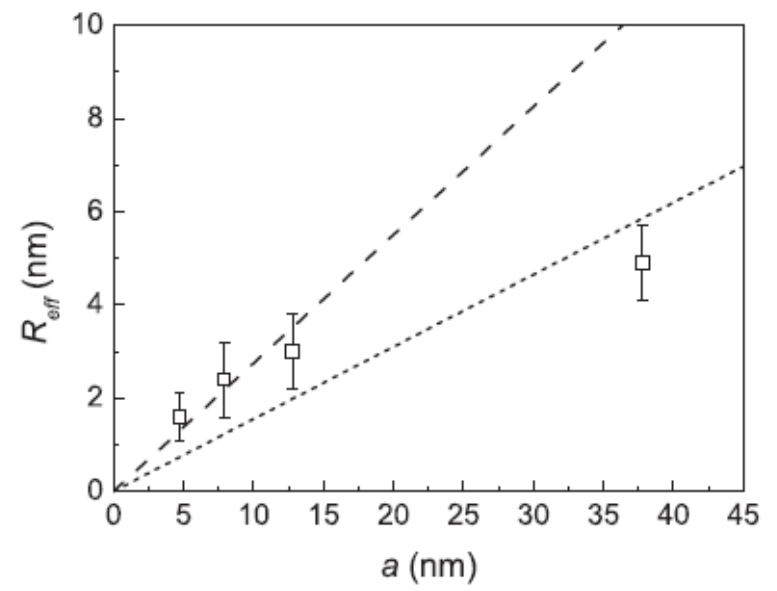

Fig. 7. Effective pore radius as a function of particle size. For comparison, the straight lines show theoretical curves calculated for random (long dash) and hexagonal (short dash) packings.
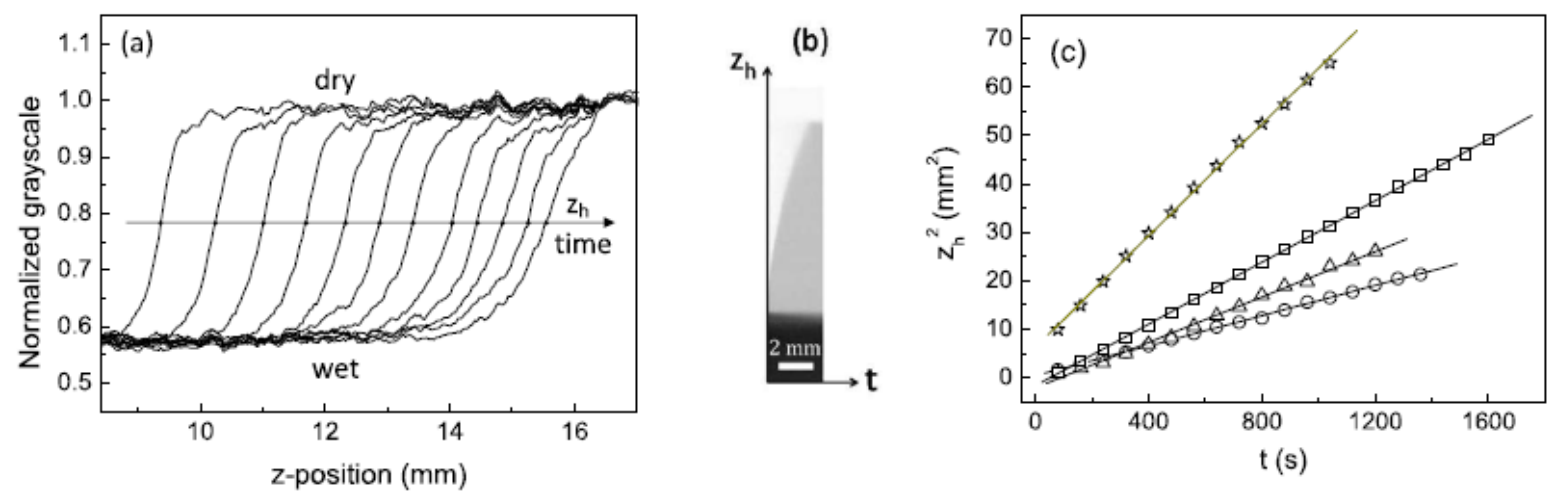

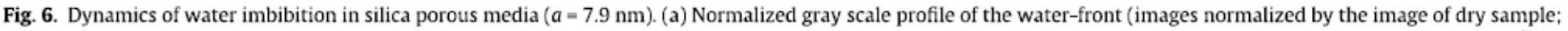

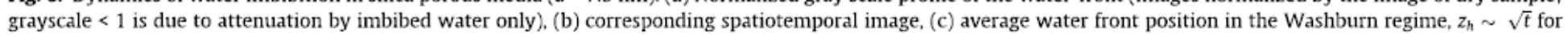
different particle sizes: $4.7 \mathrm{~nm}$ (circles), $7.9 \mathrm{~nm}$ (triangle), $12.8 \mathrm{~nm}$ (square), $37.8 \mathrm{~nm}$ (star); the solid line shows the linear fit. 
Table 3

Pore characteristics of solid gels prepared from colloidal silica of different particle sizes.

\begin{tabular}{|c|c|c|c|c|c|c|c|}
\hline$a(\mathrm{~nm})$ & $\phi_{\text {pore }, e f f}$ & $\tau$ & $R_{W}(\mathrm{~nm})$ & $R_{\text {eff }}(\mathrm{nm})$ & $R_{\mathrm{eff}} / a$ & $\kappa_{K-C}\left(\mathrm{~m}^{2}\right)$ & $\kappa_{\text {Bear }}\left(\mathrm{m}^{2}\right)$ \\
\hline 4.7 & $0.33 \pm 0.02$ & $1.74 \pm 0.04$ & $0.5 \pm 0.02$ & $1.6 \pm 0.02$ & $0.34 \pm 0.02$ & $1.9 \pm 0.05 \times 10^{-20}$ & $2.0 \pm 0.05 \times 10^{-20}$ \\
\hline 7.9 & $0.31 \pm 0.03$ & $1.80 \pm 0.05$ & $0.7 \pm 0.02$ & $2.4 \pm 0.02$ & $0.30 \pm 0.02$ & $4.0 \pm 0.05 \times 10^{-20}$ & $4.1 \pm 0.05 \times 10^{-20}$ \\
\hline 12.8 & $0.29 \pm 0.03$ & $1.86 \pm 0.05$ & $0.9 \pm 0.03$ & $3.0 \pm 0.03$ & $0.24 \pm 0.03$ & $7.9 \pm 0.06 \times 10^{-20}$ & $5.9 \pm 0.06 \times 10^{-20}$ \\
\hline 37.8 & $0.32 \pm 0.04$ & $1.77 \pm 0.07$ & $1.6 \pm 0.04$ & $4.9 \pm 0.04$ & $0.13 \pm 0.04$ & $1.1 \pm 0.07 \times 10^{-18}$ & $1.8 \pm 0.07 \times 10^{-19}$ \\
\hline
\end{tabular}

ular packings $[35,36]$ are also shown. These results show that for the porous media prepared from Ludox silica for $a=4.7$ to $12.8 \mathrm{~nm}$, the dependence of $R_{\text {eff }}$ on $a$ exhibits a behavior that falls in the range for mean random packing. For the largest size $a=37.8 \mathrm{~nm}$ however, the behavior approaches a near-hexagonal packing. This evolution in pore structure with particle size is consistent with small-angle scattering results of concentrated dispersions that show increasingly well-defined structure peaks that indicate increased particle ordering with size, with a transition to wellordered crystalline packing for $a=37.8 \mathrm{~nm}$ (see Supporting Material).

Spontaneous imbibition of water in the silica porous media is thus shown to follow the Lucas-Washburn $\sqrt{t}$ law. This capillary rise dynamics is governed by an ensemble of complex pore characteristics - grain size, effective pore radius and porosity, tortuosity that collectively define the permeability of the porous media, $\kappa$. Two common expressions relate these parameters. The first is the classic Kozeny-Carman relation for stacked spheres of radius a: [37]

$\kappa_{\text {Kozeny-Carman }}=\frac{1}{6 \tau} \frac{\phi_{\text {poreefff }}^{3}}{\left(1-\phi_{\text {pore }, \text { fff }}\right)^{2}} \frac{a^{2}}{9}$

The second, proposed by Bear, expresses permeability for an isotropic porous media with three parallel capillary networks in terms of the effective pore radius: [38]

$\kappa_{\text {Bear }}=\frac{1}{24} \frac{\phi_{\text {pore }, \text { ff } R_{\text {eff }}^{2}}}{\tau}$

The permeabilities evaluated from these two expressions using the experimentally determined values for $\phi_{\text {pore,eff. }} R_{\text {eff }}$ and $\tau$, are given in Table 3 for the silica gels of different particle sizes. We find that the two expressions give results that are close to each other for the smaller particles and fall in the range of permeability values for porous media of packed spheres [7]. With increase in particle size however, the values from the two expressions diverge. $\kappa_{\text {Bear }}$ relies on a model of parallel isotropic capillary network, and since the pore structure tends towards increasingly organized sphere packing behavior with size, the Bear model may be less suitable to describe these larger-particle systems.

\subsection{Anomalous liquid front broadening}

The liquid-front $z_{h}$ extracted from the dynamic measurements represents the average position of the wet and dry interface. An additional and informative feature in solvent imbibition concerns the width of this propagating liquid front. In real porous media, due to its disordered nature, this interface is characterized by an associated roughness, the width of which also evolves with time. In spontaneous imbibition, due to Lucas-Washburn $\sqrt{t}$ behavior the source of this interfacial roughening is attributed mainly to capillary disorder. Accordingly, the time-dependent interface width $\sigma(t)$ follows a power-law [8]:

$\sigma(t)=c t^{\beta}$

where $\beta$ is the roughening exponent and $c$ a prefactor. For laterally interconnected pores with low aspect ratio, lateral variations in liquid menisci are smoothed out by a uniform effective surface tension. As a consequence, the width of the moving front is minimized and its broadening slowed down. In this case, phase-field models predict a roughening exponent [39], $\beta=0.19$. For the other extreme of completely independent cylindrical pores of constant radii, $\beta=0.5$ because each meniscus moves independently of other pores. The roughening exponent $\beta$ therefore is an interesting parameter that relates connectivity of the liquid meniscus as the liquid advances, and thus the pore structure. It is also a relatively less often reported parameter, probably due to limited spatial resolution in large thick samples. In neutron imaging, this interfacial width can be analyzed quantitatively from the greyscale line profile along the vertical $z$-direction across the liquid front. Fig. 8 illustrates the greyscale line profile for a fragment sample at different times of imbibition. It can be seen that as imbibition progresses with time, the width of the interface increases. To quantify the width of this

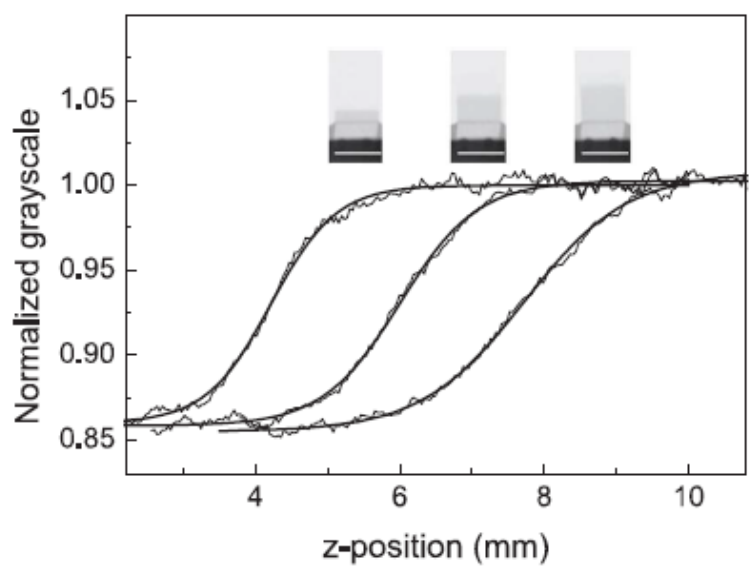

Fig. 8. Evolution of water-front width $\sigma$, during imbibition: from left to right, $5 \mathrm{~min}$, $15 \mathrm{~min}, 30 \mathrm{~min}$; the profiles are fitted to a Gaussian error function (smooth curves) with $\sigma=0.45,0.53$ and $0.71 \mathrm{~mm}$ respectively. Top images show corresponding normalized transmission images of the water-front for a fragmented sample $(a=4.7 \mathrm{~nm})$, bar scale $=1 \mathrm{~mm}$.

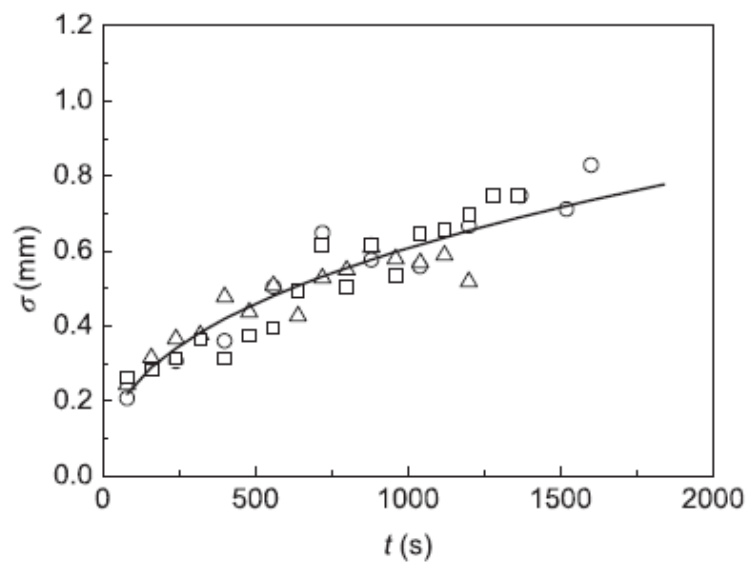

Fig. 9. Time-evolution of the water-front width $\sigma$, during imbibition in silica porous media prepared from different particle sizes: $a=4.7 \mathrm{~nm}$ (circle), $7.9 \mathrm{~nm}$ (triangle), $12.8 \mathrm{~nm}$ (square). The continuous line is a power-law fit, $\sigma(t)=c t^{\beta}$ with broadening exponent $\beta=0.41 \pm 0.01$. 
liquid front, the greyscale line profile is fitted with a Gaussian error function typically used to describe interfacial roughness:

$$
y=\frac{1}{2}\left[1-\operatorname{erf}\left(\frac{z-z_{h}}{\sqrt{2} \sigma}\right)\right]
$$

$y$ depicts the greyscale, $z_{h}$ the average liquid front position and $\sigma$ the width of the interface.

The fitted values of $\sigma$ at different imbibition times for the Ludox samples of different particle sizes are shown in Fig. 9. Separate fits to the time-evolution of $\sigma$ for each particle size yield exponent values: $\beta=0.42 \pm 0.01(4.7 \mathrm{~nm}), 0.40 \pm 0.01(7.9 \mathrm{~nm}), 0.41 \pm 0.01$ $(12.8 \mathrm{~nm})$ and $c=0.035 \pm 0.004$. No size dependence of the roughening exponent is observed for the particle sizes considered here, therefore for statistical purposes, all the data points are fitted together in a single curve. The solid line shows the best-fit curve to the ensemble of the data points for the three samples, with $\beta=0.41 \pm 0.01$ and $c=0.035 \pm 0.002 \mathrm{~mm}$. The exponent obtained for these samples indicates liquid front broadening that has also been reported in the literature. Leoni et al. [40] reported a value close to 0.5 for imbibition of a gas in disordered porous solids, where adsorption and wetting effects were invoked to explain the broadening front. Gruener et al [8] obtained $\beta=0.46$ for water imbibition in nanoporous Vycor glass, a silica system composed of elongated pores with an average radius of $4 \mathrm{~nm}$ and polydispersity $20 \%$. They attributed this broadening to the elongated structure of the pores where despite their connectivity, the menisci in the elongated pores propagate in a non-correlated manner, resulting in an anomalous broadening of the liquid front as imbibition progresses. For our current hydrophilic silica system that contains significant residual hydration water, wetting is not expected to pose a problem during the spontaneous imbibition. It is more likely that these silica gels resemble the Vycor system, where liquid front broadening during imbibition is engendered by asymmetric pores with reduced correlated menisci.

\section{Conclusions}

We have applied neutron imaging to explore, for the first time, structural properties and water imbibition dynamics in nanoporous gels prepared by controlled drying of colloidal silica dispersions of different nanometric sizes. The strong attenuation by hydrogen and the transparency of silica to neutrons render neutron imaging a unique technique to study hydrogen-rich liquids such as water in silica systems. In this case, the water content in silica porous media can be evaluated and quantified directly from transmission measurements. The main findings of our study are summarized as follows:

(1) From static transmission measurements and quantitative evaluation of the water content in the solid gels, three types of porosities have been determined: total porosity $\left(\phi_{\text {pore,tot }}\right)$ that describes all volume not occupied by the silica, effective porosity $\left(\phi_{\text {pore eff }}\right)$ that constitutes the pore volume associated with liquid imbibition, and residual porosity ( $\left.\phi_{\text {poreres }}\right)$ that accounts for the space occupied by residual chemically-bound or trapped water. Our studies show that as much as $8-20 \%$ in volume of residual water can be retained in the gel, the amount increasing with decrease in particle size. Structurally, both neutron imaging and smallangle neutron scattering (Supporting Material and from our past studies [23]) indicate an increase in particle ordering with increase in particle size. The link between residual water and particle size appears to affect the mechanical properties of the porous media [24]. Macroscopically, solid gels formed from smaller particles (higher residual water) show higher mechanical resistance compared to the more brittle gels prepared from larger particles (lower residual water). The role of residual water on material properties clearly needs further studies. Residual water in porous media has received little attention in the past, even though it constitutes an important and integral component of hydrophilic materials, as shown by our studies. To our knowledge, this neutron imaging study reports the first direct and quantitative measure of residual water in silica porous media. Compared to other techniques that probe liquid in porous media, NMR imaging has been shown [41] to be a powerful technique to probe dynamics of liquid flow in porous media. However, these techniques are adapted to probe relative changes in liquid content due to imbibition and are not sensitive to static residual water.

(2) The dynamics of spontaneous imbibition of water in the porous silica gel has been visualized in real-time by dynamic transmission measurements. Over the timescale of the experiment and for all the samples investigated, the average waterfront propagates as $\sqrt{t}$, in accordance to the LucasWashburn law. From the dynamics of imbibition, the effective pore radius $R_{\text {eff }}$ is extracted using the value of tortuosity $\tau$ evaluated from $\phi_{\text {pore eff }}$. It is found that for $a=4.7-12.8 \mathrm{~nm}$, $R_{\text {eff }}$ increases with particle size in a manner predicted for mean random packing; for the largest particle $a=37.8 \mathrm{~nm}$ however, the behavior approaches a near-hexagonal packing. This evolution in pore structure with particle size is consistent with small-angle scattering results of concentrated dispersions that show increasingly well-defined structure peaks, indicating increased particle ordering with size and a transition to well-ordered crystalline packing for $a=37.8 \mathrm{~nm}$. Surprisingly, despite the different degrees of residual water and particle ordering, the effective porosities that participate in liquid imbibition remain similar in all cases, $\phi_{\text {pore eff }} \approx 0.3$. These porosity values and the corresponding intrinsic permeabilities fall in the range reported in the literature for porous glass systems with pore size ranging from $0.4 \mathrm{~nm}$ to $1 \mu \mathrm{m}$ [7].

(3) A notable feature revealed from the real-time imbibition measurements concerns the evolution of the width of the liquid front. Although theoretical treatments of liquid front width exist in the literature [39], few experimental measurements have been reported due to limited beam penetration and insufficient spatial resolution. Neutron imaging, with the deep penetration power of neutrons, is a welladapted technique to address to this problem. It has been applied by Gruener et al [8] to study anomalous front broadening in Vycor glass with elongated pores. For our current system, quantitative analysis of the liquid front profile shows that the width of the liquid front increases with imbibition time with a broadening exponent, $\beta=0.41 \pm 0.01$. This value is larger than that predicted for an interface with correlated meniscus, and for these silica gels, may be attributed to a system of asymmetric pores with reduced correlated menisci, such as that reported in Vycor glass by Gruener et al. The liquid front broadening (or interfacial roughness) revealed in this study, although less studied experimentally, has important significance in processes such as fluid flow, mixing in fluid phases, and in adhesion.

This study presents an alternative family of silica-based nanoporous media consolidated by controlled evaporative drying. These solid gels show similar porosity and permeability properties as in other synthetic glass porous media. However, the detailed structural properties and flow behavior obtained from neutron 
techniques have further revealed new insights on the effects of particle size on residual water content and particle organization, aspects that have direct consequences on macroscopic mechanical properties of the gel. These findings open new directions to understand structure-property relationship in gels by tuning physicochemical properties that control particle interactions and organizations, and to develop new materials. The little-studied anomalous front-broadening revealed in this work can be explored in the context of mixing and adhesion. Finally, our past studies have shown that these gels exhibit mechanical properties similar to those in real systems, such as crack patterns that bear great resemblance to those produced in historical art paintings [24]. They can therefore serve as excellent model systems to understand mechanical processes in practical porous systems.

\section{CRediT authorship contribution statement}

Marguerite Léang: Methodology, Investigation, Software, Visualization. Frédéric Ott: Conceptualization, Methodology, Software. Frédérique Giorgiutti-Dauphiné: Resources, Supervision. Ludovic Pauchard: Resources, Supervision, Writing - review \& editing. Lay-Theng Lee: Conceptualization, Investigation, Supervision, Writing - original draft.

\section{Declaration of Competing Interest}

The authors declare that they have no known competing financial interests or personal relationships that could have appeared to influence the work reported in this paper.

\section{Acknowledgements}

We thank Fabrice Cousin for help in the small angle neutron scattering experiments carried out at the ORPHEE reactor at the Laboratoire Léon Brillouin. Funding Sources: This work was supported by the "Investissements d'Avenir" LabEx PALM (Grant Number: ANR-10-LABX-0039-PALM).

\section{Appendix A. Supplementary material}

Supplementary data to this article can be found online at https://doi.org/10.1016/j.jcis.2020.01.052

\section{References}

[1] M.G. Basavaraj, G.S. Gupta, New calibration technique for X-ray absorption in single and multiphase flow in packed beds, Iron Steel Inst. Jpn. Int. 44 (2004) $50-58$.

[2] M. Scheel, R. Seemann, M. Brinkmann, M. Di Michiel, A. Sheppard, B. Breidenbach, S. Herminghaus, Morphological clues to wet granular pile stability, Nat. Mater, 7 (2008) 189-193.

[3] N. Lu, B.D. Zeidman, M.T. Lusk, C.S. Willson, D.T. Wu, A Monte Carlo paradigm for capillarity in porous media, Geophys. Res. Lett. 37 (2010) L23402-L23407.

[4] M. Porus, C. Labbez, P. Maroni, M. Borkovec, Adsorption of monovalent and divalent cations on planar water-silica interfaces studied by optical reflectivity and Monte Carlo simulations, J. Chem. Phys. 135 (2011). 064701(1-9).

[5] M.E.J. Haagh, I. Siretanu, M.H.G. Duits, Mugele, Salinity-dependent contact angle alteration in oil/brine/silicate systems: the critical role of divalent cations, Langmuir 33 (2017) 3349-3357.

[6] I.S. Anderson, R.L. McGreevy, H.Z. Bilheux, Neutron Imaging and Applications: A Reference for the Imaging Community, Springer, New York, 2009.

[7] E. Perfect, C.L. Cheng, M. Kang, H.Z. Bilheux, J.M. Lamanna, M.J. Gragg, D.M. Wright, Neutron imaging of hydrogen-rich fluids in geomaterials and engineered porous media: a review, Earth Sci. Rev. 129 (2014) 120-135.

[8] S. Gruener, Z. Sadjadi, H.E. Hermes, A.V. Kityk, K. Knorr, S.U. Egelhaaf, H. Rieger, P. Huber, Anomalous front broadening during spontaneous imbibition in a matrix with elongated pores, Proc. Natl. Acad. Sci. 109 (2012) 10245-10250.

[9] S. Gruener, H.E. Hermes, B. Schillingerd, S.U. Egelhaaf, P. Huber, Capillary rise dynamics of liquid hydrocarbons in mesoporous silica as explored by gravimetry, optical and neutron imaging: nano-rheology and determination of pore size distributions from the shape of imbibition fronts, Coll. Surf. A 496 (2016) 13-27.
[10] M.R. Deinert, J.-Y. Parlange, T. Steenhuis, J. Throop, K. Ünlü, K.B. Cady Measurement of fluid contents and wetting front profiles by real-time neutron radiography, J. Hydrol. 290 (2004) 192-201.

[11] F.H. Kim, D. Penumadu, D.S. Hussey, Water distribution variation in partially saturated granular materials using neutron imaging, J. Geotech. Geoenviron. Eng. 138 (2012) 147-154.

[12] S. Raneri, G. Barone, P. Mazzoleni, E. Rabot, Visualization and quantification of weathering effects on capillary water uptake of natural building stones by neutron imaging, Appl. Phys. A 122 (2016) 969-977.

[13] C.-L. Cheng, E. Perfect, B. Donnelly, H.Z. Bilheux, A.S. Tremsin, L.D. McKay, V.H DiStefano, J.C. Cai, L.J. Santodonato, Rapid imbibition of water in fractures within unsaturated sedimentary rock, Adv. Water Resour. 77 (2015) 82-89.

[14] P. Zhang, F.H. Wittmann, T-J, Zhao, E.H. Lehmann, P. Vontobel, Neutron radiography, a powerful method to determine time-dependent moisture distributions in concrete, Nucl. Eng. Design 241 (2011) 4758-4766.

[15] R. Hassanein, H.O. Meyer, A. Carminati, M. Estermann, E. Lehmann, P. Vontobel, Investigation of water imbibition in porous stone by thermal neutron radiography, J. Phys. D Appl. Phys. 39 (2006) 4284-8291.

[16] M. Arif, D.S. Hussey, D.L. Jacobson, Neutron imaging for the hydrogen economy, in: I.S. Anderson, R.L. McGreevy, H.Z. Bilheux (Eds.), Neutron Imaging and Applications: A Reference for the Imaging Community. Springer, New York, 2009.

[17] T.A. Trabold, J.P. Owejan, JJ Gagliardo, D.L. Jacobson, D.S. Hussey, M. Arif, Use of neutron imaging for proton exchange membrane fuel cell, in: W. Vielstich, H.A. Gasteiger, H. Yokokawa (Eds.), Handbook of Fuel Cells, Wiley, West Sussex, England, 2009.

[18] T.M. Nakanishi, Neutron imaging applied to plant physiology, in: I.S. Anderson, R.L. McGreevy, H.Z. Bilheux (Eds.), Neutron Imaging and Applications: A Reference for the Imaging Community, Springer, New York, 2009.

[19] N. Kardjilov, G. Festa (Eds.), Neutron Methods for Archaeology and Cultura Heritage, Springer International Publishing AG, Cham, Switzerland, 2017

[20] C. Andreani, G. Gorini, T. Materna, Novel neutron imaging techniques for cultural heritage objects, in: I.S. Anderson, R.L. McGreevy, H.Z. Bilheux (Eds.) Neutron Imaging and Applications: A Reference for the Imaging Community, Springer, New York, 2009.

[21] Z. Kis, F. Sciarretta, L. Szentmiklosi, Water uptake experiments of historic construction materials from Venice by neutron imaging and PGAI methods, Mater. \& Struct. 50 (2017) 159-172.

[22] J.J. Boon, R. Hendrickx, G. Eijkel, I. Cerjak, A. Kaestner, E.S.B. Ferreira, Neutron radiography for the study of water uptake in painting canvases and preparation layer, Appl. Phys. A 121 (2015) 837-847.

[23] M. Léang. D. Lairez, F. Cousin, F. Giorgiutti-Dauphiné, L. Pauchard, L.-T. Lee, Structuration of the surface layer during drying of colloidal dispersions, Langmuir 35 (2019) 2692-2701

[24] M. Léang, F. Giorgiutti-Dauphiné, L.-T. Lee, L. Pauchard, Crack opening : from colloidal systems to paintings, Soft Matter 13 (2017) 5802-5808.

[25] M. Léang, L. Pauchard, L.-T. Lee, F. Giorgiutti-Dauphiné, Imbibition on a porous layer: dynamical and mechanical characterization, Soft Matter 15 (2019) 2277-2283

[26] W. Treimer, Neutron tomography, in: I.S. Anderson, R.L. McGreevy, H.Z. Bilheux (Eds.), Neutron Imaging and Applications: A Reference for the Imaging Community, Springer, New York, 2009.

[27] B.D. Cullity, Elements of X-ray Diffraction, Addison-Wesley Publishing Co., Inc. Massachusetts, USA, 1956.

[28] L. Koester, W.B. Yelon, Summary of low energy scattering lengths and cross sections, Neutron diffraction newsletter, International Union of Crystallography, Chester, England, 1983.

[29] R. Lucas, Rate of capillary ascension of liquids, Kolloid Z. 23 (1918) 15-22.

[30] E.W. Washburn, The dynamics of capillary flow, Phys. Rev. 17 (1921) 273-283.

[31] J. Bico, Mécanismes d'imprégnation: Surfaces texturées, Bigouttes, Poreux. Doctoral Thesis, l'Université de Paris VI, 2000.

[32] F.A.L. Dullien, Prediction of "Tortuosity Factors" from Pore Structure Data, AIChE J. 21 (1975) 420-422

[33] S. Carbonell, M.J. Hey, J.R. Litchell, C.J. Roberts, J. Hipkiss, J. Vercauteren Capillary flow and rheology measurements on chocolate crumb/sunflower oi mixtures, J. Food Sci. 69 (2004) E465-E470.

[34] P.W.J. Glover, E. Walker, Grain-size to effective pore-size transformation derived from electrokinetic theory, Geophysics 74 (2009) E17-E29.

[35] G. Mason. A model of the pore space in a random packing of equal spheres, ] Coll. Interf. Sci. 35 (1971) 279-287.

[36] J.D.F. Ramsay, B.O. Booth, Determination of structure in oxide sols and gels from neutron scattering and nitrogen adsorption measurements, J. Chem. Soc. Faraday Trans. I (79) (1983) 173-184.

[37] P.C. Carman, Fluid flow through granular beds, Trans. Inst. Chem. Eng. 15 (1937) $150=166$

[38] J. Bear, Dynamics of Fluids in Porous Media, American Elsevier Publishing Company, New York, 1972

[39] M. Dubé, C. Daneault, V. Vuorinen, M. Alava, M. Rost, Front roughening in three-dimensional imbibition, Eur. Phys. J. B 56 (2007) 15-26.

[40] F. Leoni, E. Kierlik, M.L. Rosinberg, G. Tarjus, Spontaneous imbibition in disordered porous solids: a theoretical study of helium in silica aerogels. Langmuir 27 (2011) 8160-8170.

[41] N. Ben Abdelouahab, A. Gossard, C. Marlière, P. Faure, S. Rodts, P. Coussot, Controlled imbibition in a porous medium from a soft wet material (poultice), Soft Matter 15 (2019) 6732-6741. 